\section{Correct Antemortem Diagnosis of Pneumonia in Children With Fatal Illnesses}

This retrospective study analyzed the level of concordance between clinical and autopsy diagnosis of pneumonia over a 3year period. Utilizing the Goldman classification, the concordance rate was found to be $37.5 \%$. Major discrepancies (Class I and II) were found in $25 \%$ cases, and minor discrepancies (Class III and IV) in $37.5 \%$ cases.

Keywords: Autopsy, Medical audit, Risk factors, Viral pneumonia.

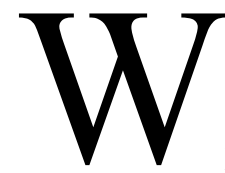

e conducted a retrospective study to analyze the level of concordance between clinical and autopsy diagnosis of pneumonia in children. A secondary objective of the study was to assess if known risk factors associated with mortality due to pneumonia were mentioned in their clinical case records.

Over a 3-year period (January, 2012 to December, 2014), 88 children were confirmed to have pneumonia on autopsy study. Their median (IQR) age was 10.5 (45) months (49 males). Detailed histopathological examination identified etiology of the pneumonia as viral in $42(47.7 \%)$, bacterial in $27(30.7 \%)$, bacterial and viral in $18(20.4 \%)$, and fungal in one (1.1\%) child.

Utilizing the Goldman classification [1], our analyses revealed that in only $33(37.5 \%)$ cases the ante mortem diagnosis of pneumonia (including its etiology) was also confirmed on autopsy to be directly related to death (Class V). In only one child the discrepancy was major (Class I) wherein had the correct diagnosis been made clinically, it would have changed patient management and might have resulted in cure or prolonged survival. This case was a 1-year-old girl with severe acute malnutrition admitted with history of fever for 21 days. Her chest radiograph revealed a bronchopneumonia which was treated with broad-spectrum antibiotics. She died after a 7.5 day stay in hospital in spite of intensive care and mechanical ventilator support. Her post-mortem examination revealed fungal (Aspergillus) bronchopneumonia.

In $21(23.9 \%)$ cases, the discrepancy was major (Class II) but the missed diagnosis of pneumonia would not have altered treatment or survival. Of these 21 cases, in 18
(20.4\%) cases the patient had already been empirically started on oral antibiotics by a private practitioner (with or without oseltamivir) for a few days before being referred to our institute. The remaining 3 (3.4\%) cases had been brought directly to our emergency department. All 21 cases were critically ill and received appropriate resuscitative management, but had succumbed before a chest radiograph could be done.

In $31(35.2 \%)$ cases, the discrepancy was minor (Class III) and the missed diagnosis of pneumonia was not directly related to death but related to the terminal disease process (e.g. septicemia, meningitis, congenital heart disease). In two (2.3\%) cases, the discrepancy was minor (Class IV) and the missed diagnosis of pneumonia was not directly related to death nor to the terminal disease process (e.g. fulminant hepatitis, leukemia, encephalitis).

Known risk factors associated with fatal outcomes of childhood pneumonia observed in majority of our cases included: age less than 1 year in $49(55.7 \%)$ [2-4], moderate and severe acute malnutrition in 55 (62.5\%) [3-5], belonging to lower socioeconomic status in $70(79.5 \%)$ [5-6], and prior complaints before hospitalization of inability to feed in $58(65.9 \%)$ [2], and altered sensorium in $55(62.5 \%)$ [4]. Small sample size precluded subgroup analysis of the data.

Our study reaffirms the importance of autopsy in hospital practice. Risk factors observed may help identify cases of pneumonia with a predilection for a poor outcome.

Contributors: SK, PV, PS: involved in study design and implementation; LK, PS: collected data; SK, PV, LK, PS: discussed core ideas and interpretation of data; SK: searched the literature and drafted the manuscript; PV, LK, PS: critically reviewed the manuscript; $\mathrm{SK}$ : will act as guarantor for this paper. All authors have approved the final manuscript.

Funding: None; Competing interest: None stated.

$$
\begin{aligned}
& \text { Sunil Karande }{ }^{1}{ }^{*} \text {, Pradeep Vaideeswar }{ }^{2} \text {, } \\
& \text { Lamk Kadiyani }{ }^{1} \text { and Pragati Sathe }{ }^{2} \\
& \text { Departments of }{ }^{1} \text { Pediatrics and }{ }^{2} \text { Pathology, } \\
& \text { Seth GS Medical College and } \\
& \text { KEM Hospital, Parel, Mumbai, India. } \\
& \text { *karandesunil@yahoo.com }
\end{aligned}
$$

\section{REFERENCES}

1. Goldman L, Sayson R, Robbins S, Cohn LH, Bettmann M, Weisberg M. The value of the autopsy in three medical eras. N Engl J Med. 1983;308:1000-5.

2. Sehgal V, Sethi GR, Sachdev HP, Satyanarayana L. 
Predictors of mortality in subjects hospitalized with acute lower respiratory tract infections. Indian Pediatr. 1997;34:213-9.

3 Lupisan SP, Ruutu P, Erma Abucejo-Ladesma P, Quiambao BP, Gozum L, Sombrero LT, et al. Predictors of death from severe pneumonia among children 2-59 months old hospitalized in Bohol, Philippines: Implications for referral criteria at a first-level health facility. Trop Med Int Health. 2007;12:962-71.

4. Dembele BPP, Kamigaki T, Dapat C, Tamaki R, Saito M, Saito M, et al. Aetiology and risks factors associated with the fatal outcomes of childhood pneumonia among hospitalised children in the Philippines from 2008 to 2016: A case series study. BMJ Open. 2019;9:e26895.

5. Tomczyk S, McCracken JP, Contreras CL, Lopez MR, Bernart C, Moir JC, et al. Factors associated with fatal cases of acute respiratory infection (ARI) among hospitalized patients in Guatemala. BMC Public Health. 2019;19:499.

6. Sharma R. Revised Kuppuswamy's socioeconomic status scale: Explained and updated. Indian Pediatr. 2017;54: 867-70.

\section{B-type Natriuretic peptide Levels and Outcome in Children With Severe Acute Malnutrition With Co-morbidity}

We studied the ability of B-type natriuretic peptide (BNP) in predicting mortality among 86 in-patients with severe acute malnutrition presenting with co-morbidity, and found that cut-off level of BNP $\geq 201 \mathrm{pg} / \mathrm{mL}$ in Receiver operating characteristics curve [AU-ROC 0.96 (95\% CI $0.92,1.003, P<0.0001)]$ had high discriminative ability to distinguish between survivors and non-survivors.

Keywords: Cardiac failure, Mortality, Under-nutrition.

Pneumonia presenting with respiratory failure is associated with heart failure, even in healthy children without cardiac risks, and high mortality [1]. However, risk of mortality and cardiac morbidity is further increased in pneumonia occurring in children with severe acute malnutrition (SAM).The accuracy of diagnosing heart failure in children with presenting with respiratory distress is difficult clinically, as signs of heart failure are subtle and mimic the features of SAM. Prior studies revealed increased levels of BNP in pneumonia complicated with heart failure which returned to lower levels with control of heart failure [2,3]. However, there is paucity of data on BNP levels in children with SAN with pneumonia. Present study, aims to identify levels BNP that predict mortality in children with SAM with comorbidities.

This case-control study was conducted from September, 2016 to May, 2018 in a tertiary care hospital in Northern India. Consecutive children, aged 6 to 60 months fulfilling the WHO criteria of SAM were enrolled as cases [4]. Age- and sex-matched children of age group
6 to 60 months with weight for length/height $>1 \mathrm{SD}$, and mid upper arm circumference $>13.5 \mathrm{~cm}$ and without pitting edema were recruited as controls from well-baby clinic of the department of pediatrics. The study was approved by the Institutional ethics committee, and informed consent was obtained from the parents before the study. Detailed history, clinical examination, socio-demographic variables, anthropometry, laboratory results, diagnosis and outcome were recorded on pre-designed form.

Co-morbidity was defined as presence of one or more additional conditions co-occurring in SAM children. Tachypnea was defined as respiratory rate $>50$ /minute in 6-12 months children and more than 40/minute in children 13-60 months. Tachycardia was defined as pulse rate $>160$ /minute in children up to one year and more than 140/ minute in children 13-60 months [4]. Clinical heart failure was defined as the presence of tachycardia, tachypnea, triple rhythm, tender hepatomegaly and engorged jugular veins [5]. Biochemically heart failure was defined as BNP levels $>300 \mathrm{pg} / \mathrm{mL}$ [6]. Investigations included arterial blood gas analysis, serum electrolytes, calcium, serum albumin, blood sugar, $X$-ray chest, and any other as per indication. The Alere Triage Cardio 3 panel was used to estimate levels of BNP in SAM children and age- and sexmatched healthy children as per manufacture's guidelines.

For sample size calculation, we measured BNP levels in 16 children with SAM and found that mean (SD) BNP level was 22.6 (25.27) and ranged from 1.8 to $103 \mathrm{pg} / \mathrm{mL}$. Considering $\leq 100 \mathrm{pg} / \mathrm{mL}$ as normal levels, and assuming $20 \%$ increase in BNP levels in SAM children with comorbidity, with power of study as $90 \%$ and with alpha error of 0.05 , a sample size of 75 SAM children with SAM was required.

Data were analyzed by using SPSS (version 16.0). The Receiver operating characteristic (ROC) curve analysis was performed to obtain the area under the curve (AUC) 